\title{
Study on time delay estimation in boiler acoustic temperature measurement based on third correlation PHAT- $\beta$
}

\author{
WANG Mianmian $^{1, *}$, LIU Wenhong ${ }^{1}$ and XU Keni ${ }^{1}$ \\ ${ }^{1}$ School of Electronic and Information, shanghai Dianji University, China
}

\begin{abstract}
In order to accurately measure the temperature of power plant boiler, a new algorithm of time delay was proposed based on third correlation and phase transform weighting on the basis of the research of traditional cross-correlation method and generalized cross-correlation. Small peaks can be weakened with the help of phase transform weighting and the addition of exponential coefficient $\beta$. The simulation result shows that the PHAT- $\beta$ algorithm based on third correlation can accurately measure the value of time delay estimation compared with first correlation, second correlation and traditional third correlation, so as to improve the accurancy of temperature of power plant boiler.
\end{abstract}

\section{Introduction}

The temperature parameters in the furnace are very important for the safe operation of large utility boilers, but it is difficult to measure because of the large volume and complex structure of the boiler. The traditional contact temperature measurement method is difficult to adapt to high temperature, corrosion, dust and other environments. As a new method, acoustic temperature measurement has the advantages of non-contact, high accuracy, real-time and so on, which has attracted wide attention in the research of boiler temperature measurement ${ }^{[1-2]}$. In some developed countries, acoustic temperature measurement has been put into practical production and life, but the technology is still not mature, there are still many problems. In China, due to the lack of research institutions and personnel, the research of acoustic temperature measurement is mainly in the laboratory and simulation stage, and there are still many problems in practical application. An Liansuo ${ }^{I_{3} l}$ and others studied the influence of sound source signal and sound source position on furnace temperature measurement. Shen Guoqing ${ }^{[4]}$ and others have carried out a series of experimental studies on time delay estimation in acoustic temperature measurement of utility boilers. By comparing various weighting functions of generalized cross-correlation, it is concluded that Phat - $\beta$ weighting has a strong ability to suppress reverberation, and ML weighting can effectively suppress noise. Li Yanqin and others [5] studied the propagation of sound wave in the furnace and reconstructed the two-dimensional velocity field in the furnace.

The key of acoustic temperature measurement is to measure the transit time of sound wave in the furnace, which is essentially a time delay estimation problem ${ }^{\text {I6- }}$
71 .Time delay estimation is an important content in the field of digital signal processing, which has important theoretical significance and practical value. It has a wide range of applications in acoustic positioning, radar, earthquake, biomedicine and so on. At present, the crosscorrelation function method is mainly used to measure the transit time of acoustic signal ${ }^{[8]}$. The traditional cross-correlation method is simple in operation, but there are many problems in accuracy. In the generalized crosscorrelation method, many generalized weighting functions are proposed to suppress noise, so as to improve the accuracy of time delay. Although time delay estimation has been fully applied in many fields, there are relatively few researches on the monitoring of boiler temperature in power plant. When using acoustics to monitor furnace temperature in electric field, workers need to measure many times to get relatively accurate data $^{[9]}$. To solve this problem, based on the study of primary correlation, secondary correlation and tertiary correlation, this paper adopts the algorithm of time delay estimation based on tertiary correlation, which uses the autocorrelation function of two input signals and the traditional cross-correlation power spectrum function to do cross-correlation, so as to increase the power spectrum function of tertiary correlation at the peak value, weaken other small peaks, and improve the accuracy of time delay estimation. It can monitor the temperature in the furnace well.

\section{Principle of acoustic temperature measurement}

The basic principle of using acoustics to measure boiler temperature ${ }^{[10]}$ is to install sound generating and receiving devices on both sides of the boiler furnace. With the help of the relationship between the 
propagation speed of sound wave in the medium and the temperature, the propagation speed of sound wave in the propagation path is obtained, so as to obtain the path temperature ${ }^{[11]}$. Taking a single path as an example, a simplified time delay estimation model is shown in Figure 1.

acoustic source
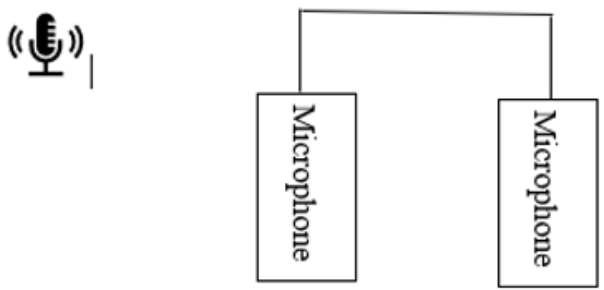

Fig.1 Time delay estimation model

The equation of acoustic temperature measurement is as follows:

$\mathrm{c}=\frac{l}{\tau}=\gamma \sqrt{\frac{R}{m}(t+273.15)}=Z \sqrt{(t+273.15)}$

Where: $c$ is the propagation velocity in the medium, $\mathrm{m} / \mathrm{s} ; l$ is the distance between the sound wave generator and the receiver, $\mathrm{m} ; \tau$ is the sound wave transit time, $\mathrm{s} ; \gamma$ is the adiabatic index of the gas (the ratio of constant pressure specific heat capacity to constant volume specific heat capacity); $R$ is the universal constant of ideal gas, $\mathrm{J} / \mathrm{mol} \cdot \mathrm{K} ; m$ is the gas component, $\mathrm{kg} / \mathrm{mol} ; t$ is the gas temperature, ${ }^{\circ} \mathrm{C}$.

For a given gas compound $Z=\sqrt{\frac{\gamma R}{m}}$ is a constant. Therefore, the calculation formula of single path temperature is as follows:

$$
t=\left(\frac{l}{\tau Z}\right)^{2}-273.15
$$

\section{Time delay estimation algorithm based on cross correlation}

\subsection{Captions/numbering}

Let the signals received by the two sensors be:

$$
\left\{\begin{array}{c}
x_{1}(n)=s(n)+n_{1}(n) \\
x_{2}(n)=\alpha s(n-D)+n_{2}(n)
\end{array}\right.
$$

Where, $x_{1}(n)$ and $x_{2}(n)$ are signals received by two microphones, $s(n)$ is the function of sound source signal, $n_{1}(n)$ and $n_{2}(n)$ are noise received by two microphones, $D$ is the time delay between two microphones, $\alpha$ is the relative attenuation coefficient of sound wave, usually 1.

The cross-correlation time delay estimation method estimates the time delay through the correlation function of two received signals. When the signal and noise are zero mean stationary random processes and independent of each other, the correlation functions of $x_{1}(n)$ and $x_{2}(n)$ in equation (3) are expressed as follows:

$$
\begin{aligned}
R_{x_{1} x_{2}}(\tau) & =E\left[x_{1}(n) x_{2}(n+\tau)\right] \\
= & E\left[\left(s(n)+n_{1}(n)\right)\left(s(n-D+\tau)+n_{2}(n+\tau)\right)\right] \\
= & E\left[s(n) s(n-D+\tau)+s(n) n_{2}(n+\tau)\right. \\
& \left.+s(n-D+\tau) n_{1}(n)+n_{1}(n) n_{2}(n+\tau)\right] \\
& =R_{s s}(\tau-D)+R_{s n_{1}}(\tau-D)+R_{s n_{2}}(\tau)+R_{n_{1} n_{2}}(\tau)
\end{aligned}
$$

Assuming that signal and noise, noise and noise are independent of each other, there are:

$$
\left\{\begin{array}{c}
R_{s n_{1}}(\tau-D)=0 \\
R_{s n_{2}}(\tau)=0 \\
R_{n_{1} n_{2}}(\tau)
\end{array}\right.
$$

At this time, formula (4) can be written as

$$
R_{x_{1} x_{2}}(\tau)=R_{s s}(\tau-D)
$$

From the properties of autocorrelation function, we can see that:

$$
\left|R_{s s}(\tau)\right| \leq R_{s s}(0)
$$

So at that time, the maximum value is taken, that is, the time point corresponding to the peak point of the autocorrelation function of two received signals is the value of time delay ${ }^{[12]}$. However, in practical application, the additive noise is not necessarily ideal Gaussian white noise, and the observed signal can not be infinite. Therefore, it is not necessarily strictly 0 , but always exists. However, because the signal and noise are usually regarded as uncorrelated, the amplitude is significantly reduced. To sum up, the signal-to-noise ratio has been greatly improved after autocorrelation processing.

\subsection{Quadratic correlation time delay estimation algorithm}

Due to the problem of time delay estimation of signal by primary correlation, Tang Juan $113 \mathrm{I}$ proposed that autocorrelation function of time delay and crosscorrelation function of time delay of secondary correlation can be used for cross-correlation again. Since correlation function is also a function of time, it can be used to replace it, and the secondary correlation function can be obtained as follows:

$$
R_{R R}(\tau)=E\left[R_{11}(n) R_{12}(n+\tau)\right]
$$

After simplification, we can get the following results:

$$
R_{R R}(\tau)=R_{R S}(\tau-D)+R_{R V}(\tau)
$$

Among them, $R_{R S}(\cdot)$ represents second correlation for pure signal, $R_{R V}(\cdot)$ represents second correlation on behalf of noise, and cross-correlation function of signal and noise is ignored. Assuming that the noise is uncorrelated Gaussian white noise, it can be regarded as 0 . 


$$
R_{R R}(\tau)=R_{R S}(\tau-D)
$$

\subsection{Generalized cross correlation}

Due to the influence of noise and reverberation, there may be a big error in using the cross-correlation function method directly. Knapp and others ${ }^{[14]}$ proposes a generalized correlation method. The essence of generalized cross-correlation is to convert the collected discrete time-domain signal to frequency-domain, calculate the power spectrum of the signal and then weight it. The function of weighting is to pre filter the signal, reduce the influence of noise on the signal, so as to improve the high-frequency component of the signal. According to Wiener schinchin theorem, the cross power spectral function and correlation function are Fourier transform pairs. The cross correlation function can be obtained by inverse Fourier transform of weighted power spectral density, and then the time delay can be estimated by peak detection. The cross power spectrum function is as follows:

$$
G_{x_{1} x_{2}}=F^{*}\left[x_{1}(n)\right] F\left[x_{2}(n)\right]
$$

Where: $F$ represents Fourier transform; ${ }^{*}$ represents conjugate.

The generalized cross-correlation function is as follows:

$$
\begin{aligned}
R_{x_{1} x_{2}}(\tau) & =F^{-1}\left[\varphi(f) G_{x_{1} x_{2}}(f)\right] \\
& =\int_{-\infty}^{\infty} \varphi(f) G_{x_{1} x_{2}}(f) e^{j 2 \pi f_{\pi}} d f
\end{aligned}
$$

Where: $F^{-1}$ represents the inverse Fourier transform; $\varphi(f)$ represents the weight function, the more commonly used weight functions are Roth weighted, scot weighted, Phat weighted, etc.

In this paper, the generalized cross-correlation Phat weighting (GCC-PHAT) is used as a reference, the final correlation function is as follows :

$$
R_{x_{1} x_{2}}(\tau)=\int_{-\infty}^{\infty} \frac{1}{\left|G_{x_{1} x_{2}}(f)\right|^{\beta}} e^{j 2 \pi f_{\pi}} d f
$$

\section{Time delay estimation method based on cubic correlation and Phat}

Based on the quadratic correlation time delay estimation, the algorithm of cubic correlation time delay estimation ${ }^{[15]}$ uses the autocorrelation power spectrum function of two input signals and the traditional cross-correlation power spectrum function to do cross-correlation. Compared with quadratic correlation, the accuracy is improved to a certain extent, but the time delay can not be well estimated when the signal-to-noise ratio is low. In this paper, a time delay estimation algorithm based on
Phat - $\beta$ of cubic correlation is proposed, the flow chart is shown in Figure 2.

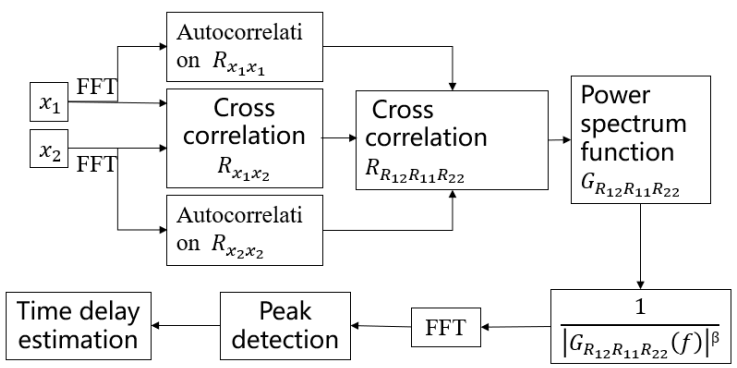

Fig.2 Block diagram of third correlation PHAT

Firstly, figure out the cross-correlation function of $x_{1}(n)$ and $x_{2}(n)$,

$$
\begin{aligned}
R_{x_{1} x_{1}}(\tau)= & E\left[x_{1}(n) x_{1}(n+\tau)\right] \\
= & R_{s s}(\tau)+R_{s n_{1}}(\tau)+R_{n_{1} s}(\tau)+R_{n_{1} n_{1}}(\tau) \\
R_{x_{2} x_{2}}(\tau) & =E\left[x_{2}(n) x_{2}(n+\tau)\right] \\
& =R_{s s}(\tau-D)+R_{s n_{2}}(\tau-D) \\
& +R_{n_{2} s}(\tau-D)+R_{n_{2} n_{2}}(\tau)
\end{aligned}
$$

Then the cross-correlation function of $x_{1}(n)$ and $x_{2}(n)$ is obtained, as shown in formula (4). And the third correlation function is obtained.

$$
R_{R_{12} R_{11} R_{22}}(\tau)=E\left[R_{12} R_{11}(n) R_{22}(n-\tau)\right]
$$

Where:

$$
\begin{aligned}
R_{12} R_{11}(n) & =E\left[R_{12}(n) R_{11}(n-\tau)\right] \\
& =\mathrm{R}_{R_{S S}}(\tau-D)+E\left[R_{s s}(n) R_{n_{1} n_{2}}(n-\tau)\right] \\
& +E\left[R_{n_{1} n_{2}}(n) R_{s s}(n-D-\tau)\right]+R_{R_{n_{1} n_{2}}}(\tau)
\end{aligned}
$$

As assumed above, we can get:

$$
R_{12} R_{11}(n)=\mathrm{R}_{R_{S S}}(\tau-D)
$$

Where: $\mathrm{R}_{R_{S S}}$ represents the cubic correlation between signals, and the time delay value $D$ is the time point corresponding to the abscissa when the correlation function peak. According to the new sequence, the cross power spectrum function $G_{R_{R_{s s}}}(f)$ can be obtained, and the selection of the weight function is particularly critical. After consulting the data, it can be seen that the generalized cross-correlation time delay based on Phat weighting is more suitable for the actual boiler temperature measurement environment. The cross power spectrum function of cubic correlation Phat algorithm is as follows :

$$
G_{R_{12} R_{11} R_{22}}=F^{*}\left[R_{12} R_{11}(n)\right] F\left[R_{22}(n)\right]
$$




\section{Computer simulation experiment}

In order to test the performance of the third correlation Phat $\beta$ algorithm, a simulation experiment of signal plus noise is constructed.

The stationary source signal is shown in Figure 3:

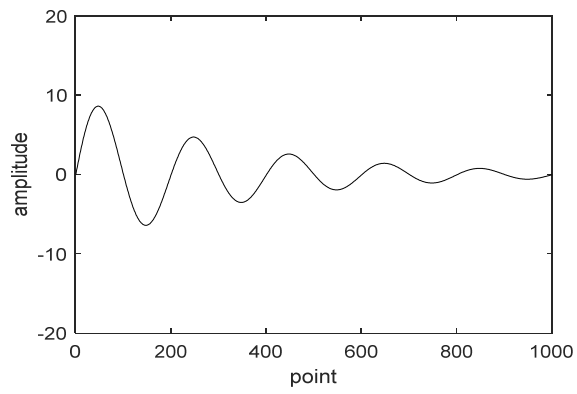

Fig.3 stationary source signal

The length of the signal is 1024 points, the sampling frequency is $1000 \mathrm{~Hz}$, and the time delay is $0.03 \mathrm{~s}$. When $\mathrm{SNR}=-10 \mathrm{~dB}$, the two signals are shown in Figure 4.
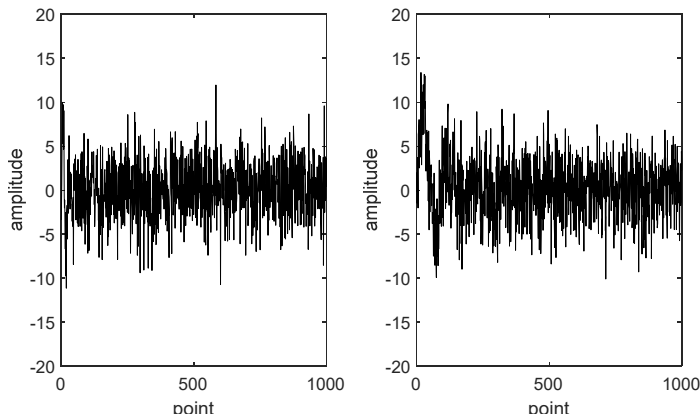

Fig.4 Noisy signal

After adding uncorrelated white noise to the signal, the simulation is carried out under different SNR. It can be seen from Fig. 5 and Fig. 6 that when SNR $=-5 \mathrm{~dB}$, the time delay can be estimated by primary correlation, secondary correlation and tertiary correlation, but the influence of secondary correlation and tertiary correlation sidelobe is greater. Therefore, when the noise is small, the time delay estimation effect of one-time correlation is better. When SNR $=-20 \mathrm{db}$, the time delay can not be estimated by primary correlation and secondary correlation, and the third correlation can still estimate the delay, and the effect is far more than primary correlation and secondary correlation.
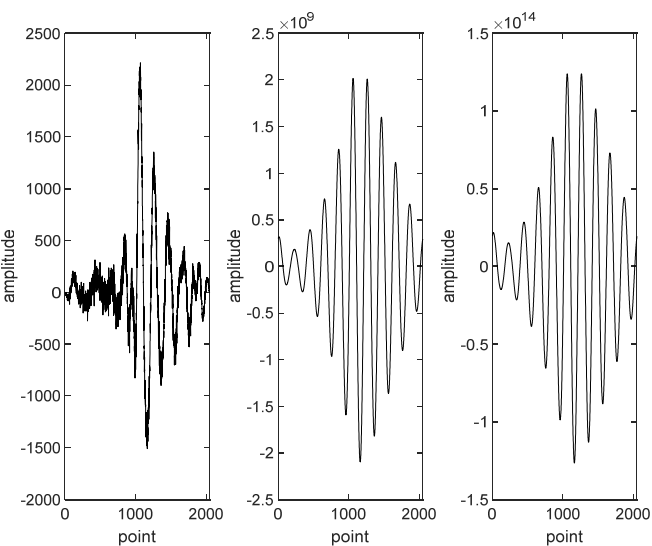

Fig.5 Uncorrelated white noise with $\mathrm{SNR}=-5 \mathrm{~dB}$
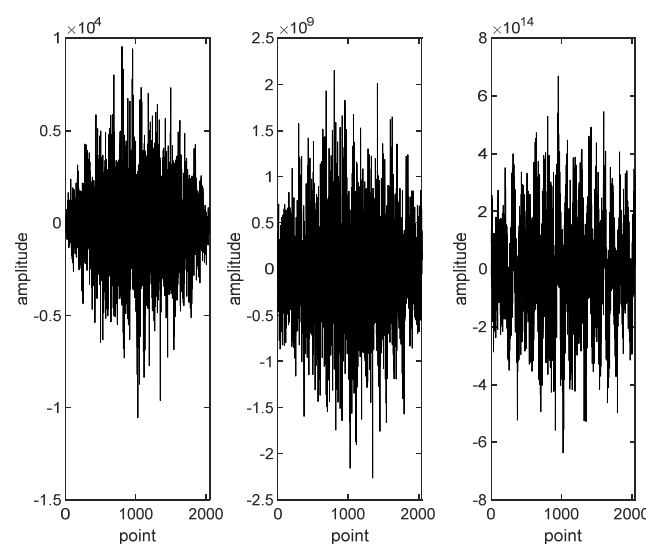

Fig.6 Uncorrelated white noise with $\mathrm{SNR}=-20 \mathrm{~dB}$

As can be seen from Fig. 6, although the time delay estimation of cubic correlation can be estimated, the peak value of side lobe is large and there are a lot of pseudo peaks. Therefore, this paper proposes a Phat algorithm based on cubic correlation. As can be seen from Fig. 7, when $\mathrm{SNR}=-5 \mathrm{~dB}$, the third correlation under Phat weighting has obvious peak value, which can better estimate the time delay. When $\mathrm{SNR}=-20 \mathrm{~dB}$, the third correlation peak under Phat weighting is also obvious. Therefore, in the case of low SNR, the third correlation under Phat weighting can effectively suppress noise and obtain more accurate time delay estimation.

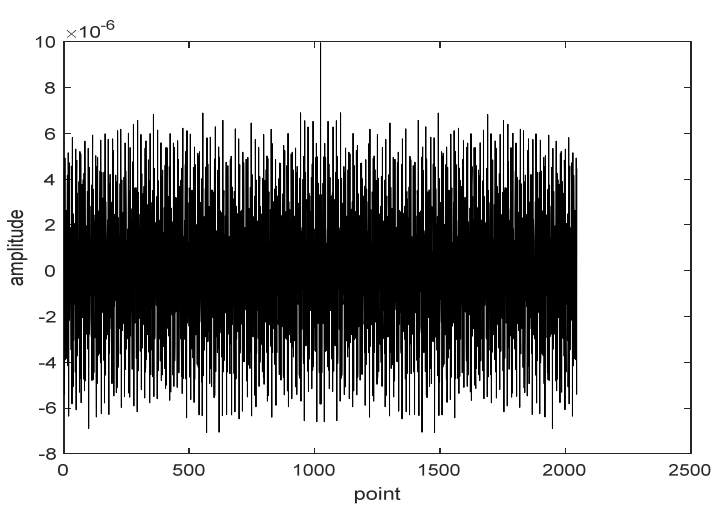

Fig.7 Third correlation under uncorrelated white noise with $\mathrm{SNR}=-5 \mathrm{~dB}$

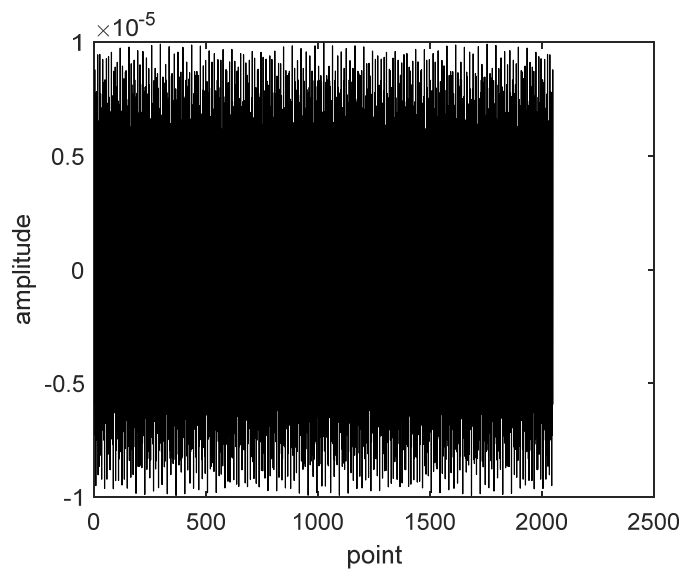

Fig.8 Third correlation under uncorrelated white noise with $\mathrm{SNR}=-20 \mathrm{~dB}$

It can be seen from figure 8 that in the low SNR environment, although the third correlation Phat 
algorithm can obtain the time delay estimation value, when the peak is amplified, it can be seen that its sidelobe has a large peak, and there are many pseudo peaks. In the process of multiple sampling, it is easy to mistake the pseudo peak as the peak, thus affecting the accuracy of time delay estimation. Therefore, based on the three-time correlation Phat algorithm, the $\beta$ parameter is introduced to effectively suppress the peak value of sidelobe, as shown in Figure 9.
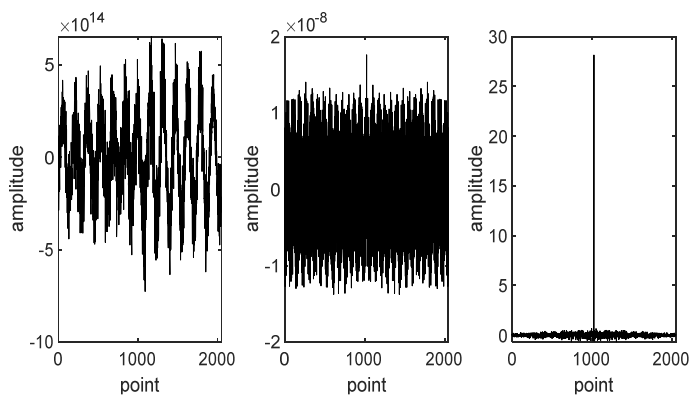

Fig.9 Uncorrelated white noise with $\mathrm{SNR}=-20 \mathrm{~dB}$

As can be seen from Figure 9, by comparison, after introducing the coefficient $\beta$, the peak value of the function is more obvious, and the influence of the sidelobe is removed. It has better anti noise performance, and can more accurately estimate the time delay value, so as to obtain more accurate boiler furnace temperature.

\section{Conclusions}

In this paper, a new method based on three correlation Phat- $\beta$ algorithm is proposed for temperature monitoring of utility boilers. In the free sound field, the time delay can be estimated by primary correlation, secondary correlation and tertiary correlation, but there are many pseudo peaks, which have a great impact on the accuracy of time delay estimation.Among them, the effect of triple correlation is better than that of primary correlation and secondary correlation, and then comparing the secondary correlation Phat algorithm with the tertiary correlation Phat algorithm, it is found that the time delay accuracy has been significantly improved. At low SNR, the performance of cubic correlation Phat algorithm is better than that of quadratic correlation Phat algorithm. Therefore, further research on the cubic correlation Phat algorithm, through continuous comparison, we find that adding exponential coefficient $\beta$ to Phat weight function can get more accurate delay estimation.

\section{References}

1. BRAMANTI M, SALERNO E A . An acoustic pyrometer system for tomographic thermal imaging in power plant boilers[J]. Instrumentation \& Measurement IEEE Transactions on, 1996, 45(1):159-167.

2. ZHANG X D,GAO B,SONG Z P.The Research of Acoustic Measuring of Gas Temperature Employ- ing Cross-Correlation Algorithm[J]. Proceedings of the CSEE,2003,23(004);185-188.

3. AN L S,SHEN G Q,ZHANG B,et al. Experiment Study on Acoustic Pyrometry in Power Plant Boiler[J]. Power System Engineering, 2007(02):2325.

4. SHEN G Q,ZHANG S P,AN L S. Study on Time Delay Es-timation in Acoustic Pyrometry Under Reverberation and High Noise Environments[J]. Journal Of Chinese Society Of Power Engineering, 2014, 34(7):529-533.

5. LI Y Q,ZHOU H C,ZHOU X H.Experimental Study on Measurement of 2-D Velocity F ILED IN a Cold Model Furance by Acoustic Method[J]. Proceedings of the CSEE, 2006, 26(013):117-122.

6. SHEN G Q,AN L S,JIANG G S,et al. Simulation of Time Delay Estimation in Acoustic Pyrometry in Power Plant Boiler[J]. Proceedings of the CSEE, 2007(11):57-61.

7. ODEH S D, MORRISON G L, BEHNIA M . Modelling of parabolic trough direct steam generation solar collectors[J]. Solar Energy, 1998, 62(6):395-406.

8. LIU L Y,TIAN F,LI Z Z,et al.Design and relization of the acoustic waves flight time measurement system based on cross-correlation method[J].Journal of Shenyang Institude of Aeronautcal Engineering,2005,22(1):57-60.

9. SHEN G Q,YANG J D,CHEN D,et al. Study on Time Delay Estimation in Boiler Acoustic Temperature Measurement Based on Second Correlation PHAT- $\beta$ Algorithm[J]. Journal Of Chinese Society Of Power Engineering, 2018, 38((08)):617-623.

10. TIAN F,SHAO F Q,WANG F L. An Introduction to Meas-uring Technique on Temperature Field for Industrial Boiler Based on Acoustic Wave[J]. Journal Of Shenyang Aero-space University, 2001, 18(3):10-11.

11. LI W N.The high precision and fast measurement method of Sound travel-time based on the crosscorrelation[D].

12. WANG H Y. Adaptive noise cancellation and time delay estimation[M]. Dalian University of Technology Press, 1999.

13. TANG J,HANG H Y. Time Delay Estimation Based on Second Correlation[J]. Computer Engineering, 2007, 33(021):265-267.

14. KNAPP C , CARTER G. The generalized correlation method for estimation of time delay[J]. IEEE Transactions on Acoustics Speech \& Signal Processing, 2003, 24(4):320-327.

15. HE W J,YAN T F,ZHANG Y, et al. Time Delay Estimation Algorithm Based on Third Correlation[J]. Journal of Lan-zhou Jiaotong Universit, 2019, 038(001):66-71. 